\title{
ARTICLE OPEN Room-temperature processing of silver submicron fiber mesh for flexible electronics
}

\author{
Xiaopeng Bai ${ }^{1}{ }^{1}$, Sen Lin ${ }^{1}$, Haolun Wang ${ }^{1}$, Yi Zong ${ }^{1}$, Haiyang Wang ${ }^{1}$, Ziyun Huang ${ }^{1}$, Donglai Li ${ }^{1}$, Chang Wang ${ }^{1}$ and Hui Wu ${ }^{1}$
}

Ultrathin, patterned, conducting metallic fibers have been extensively studied as building blocks in flexible electronics. However, their scalable processing and attainment of patterns at room temperature is challenging. In this paper, we report on the patterning of ultra-long silver submicron fibers as woven mesh through the process of continuous draw spinning in the presence of ultraviolet (UV) treatment. The silver fibers can be directly intertwined on flexible substrates, such as polyethylene terephthalate (PET) and polyimide (PI). The as obtained silver submicron fiber mesh present excellent photoelectric properties $\left(T=90 \%, R=9 \Omega \mathrm{sq}{ }^{-1}\right)$ and outstanding flexibility and can be easily transferred on other surfaces. To demonstrate its application, flexible electrochromic smart window and infrared stealth film have been prepared.

npj Flexible Electronics (2018)2:3 ; doi:10.1038/s41528-017-0016-7

\section{INTRODUCTION}

Current electronics devices are expected to be soft, flexible, even stretchable, and may be applied in non-planar substrates with topping performance. ${ }^{1-7}$ Ultrafine metal fibers are one of the key components used to prepare flexible electronics devices fulfilling the above characteristics, ${ }^{8-11}$ and they have been extensively studied in a variety of fields, such as smart windows, ${ }^{12}$ touch screen, ${ }^{13-15}$ nanocircuits, ${ }^{16-18}$ artificial electronic skin, ${ }^{19-24}$ solar cells, ${ }^{25}$ and interactive electronics. ${ }^{26}$ In the context of these applications, how to use the ultrafine metal fibers on flexible substrate is a pivotal problem. This is because the most flexible substrates are generally thermosensitive materials, while the preparation of metal fibers generally requires high-temperature treatment. Hence, obtaining highly conductive and ultrafine fibers at low/room temperature is a huge challenge. At present, production methods of ultrafine conductive fibers at room or low temperature are mainly based on chemical synthesis, ${ }^{27}$ metal nanotrough network, ${ }^{28}$ laser nano-welding process, ${ }^{29}$ supersonic spraying, ${ }^{30}$ mechanical pressing ${ }^{31}$ etc. Although these methods have their own advantages, the optoelectronic performance of as prepared conducting fibers are limited by their low conductivity, or high cost. In addition, there are no other facile methods to obtain the woven mesh of metal fibers, and the synthesis of free standing metal fiber mesh at room temperature with low cost is still a problem.

In our previous work, we have reported the continuous draw spinning technique to prepare extra-long submicron fibers with micrometer patterning capability, ${ }^{32}$ which is convenient for choosing the discretionary substrate on the basis of the requirements of users. However, the process of preparation requires sintering silver precursor fibers at $250^{\circ} \mathrm{C}$ followed by insulation for $2 \mathrm{~h}$, which is not suitable for heat-sensitive substrates. In addition, the silver precursor fiber mesh is prone to partial breakage during the process. Hence, their practical industrial application is highly limited. In this paper, we report a facile and economical way to prepare silver submicron fiber mesh, which includes two main steps: in the first step, silver precursor fibers are prepared through continuous draw spinning, in the second step, the as obtained silver precursor fiber mesh was irradiated by ultraviolet (UV) light, enabling the reduction of silver precursor to silver. The method is milder compared the hightemperature treatment process. Moreover, the polymer skeleton which are retained among silver fibers, greatly enhance their flexibility and toughness.

\section{RESULTS}

Figure 1a shows the schematic diagram depicting the synthesis process of silver submicron fiber mesh at room temperature. Firstly, silver precursor fiber is prepared through draw spinning by an equipment set designed by us, following which the obtained silver precursor fiber mesh along with an anaerote atmosphere is placed in a sealed quartz glass box and exposed to UV light. The final form of the silver fiber mesh was obtained after UV irradiation. Figure $1 \mathrm{~b}$ shows the digital photograph of silver fiber mesh, showing that it has excellent transparency and flexibility. In addition, owing to the effect of special array structure, there was no reduction in transparency by scattering of light (Fig. S1). The silver submicron fiber mesh connected to the light-emitting diode (LED) circuit is shown in Fig. 1c. Despite the bending of the fibers mesh, there is no flickering in the LED light (Supplementary Video S2), proving that the sheet resistance of the silver fiber mesh maintains a stable value during the bending process.

Figure 2a shows the scanning electron microscopy images of silver precursor fibers after being irradiated by UV light for different durations of time (from left to right: $0 \mathrm{~h}, 1 \mathrm{~h}, 3 \mathrm{~h}, 5 \mathrm{~h}$, respectively). This study is conducted for exploring the influence of irradiation time on the morphology of fibers, which indicates that the UV irradiation leads to the formation and integration of silver nanoparticles. $\mathrm{AgNO}_{3}$ acts as the silver precursor owing to its good stability in the Polyvinylpyrrolidone (PVP), the PVP is used as the carrier of the silver precursor. With the increase of irradiation

\footnotetext{
${ }^{1}$ State Key Laboratory of New Ceramics and Fine Processing, School of Materials Science and Engineering, Tsinghua University, Beijing 100084, China Correspondence: Hui Wu (huiwu@tsinghua.edu.cn)
}

Received: 28 June 2017 Revised: 4 September 2017 Accepted: 12 October 2017

Published online: 25 January 2018 
a

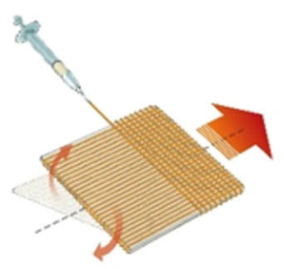

UV light

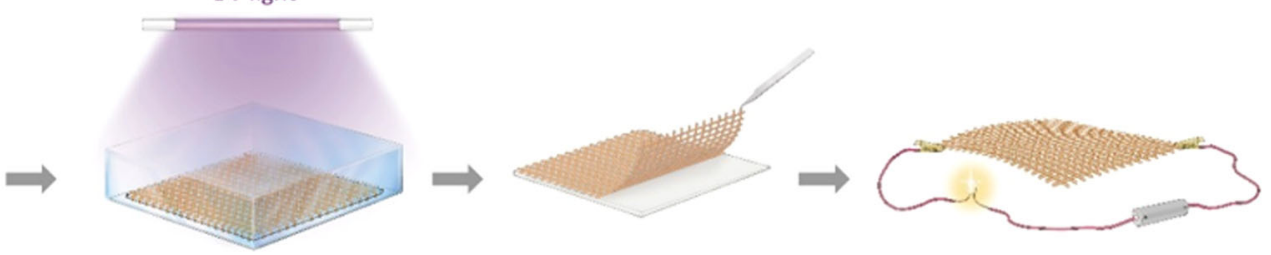

b
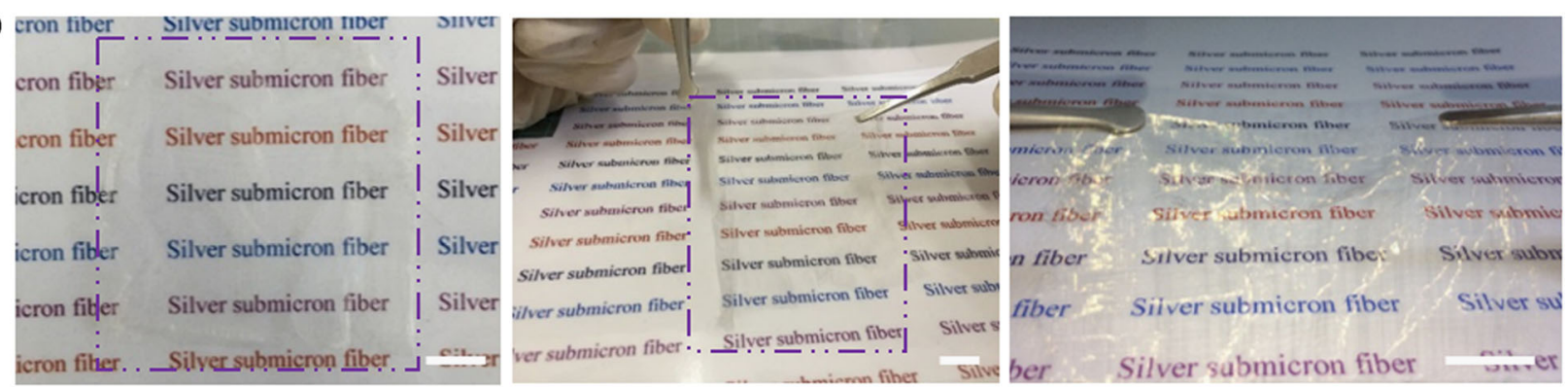

C
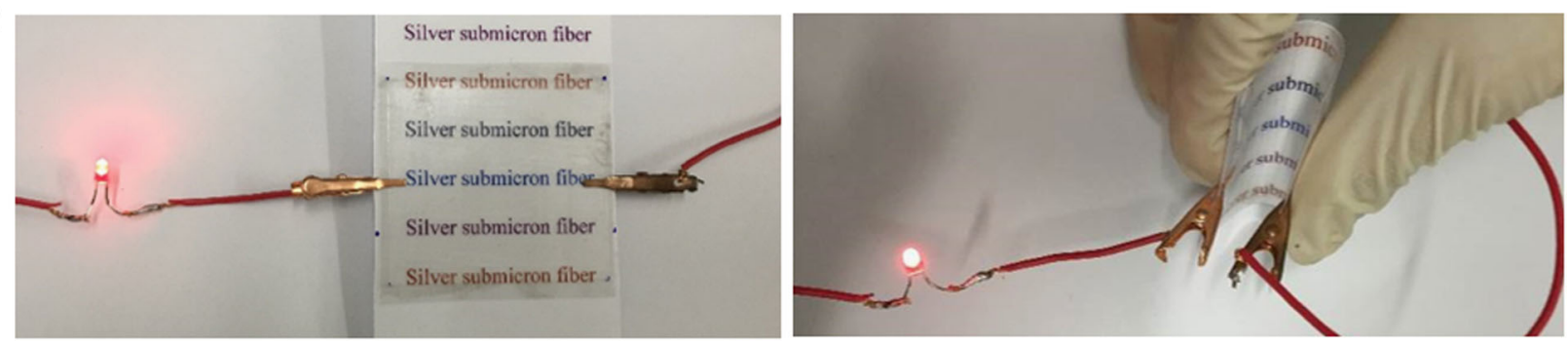

Fig. 1 Preparation process of silver submicron fiber. a Schematic diagram for preparing the silver submicron fiber at room temperature. $\mathbf{b}$ Digital photograph of silver fibers after UV radiation for $5 \mathrm{~h}$ (scale bar: $1 \mathrm{~cm}$ ), which shows that the silver fiber mesh has excellent transparency and flexibility. c Silver fiber mesh attached in the PET film that lightened on the LED lamp. (left: spread out; right: double back)

time, PVP is gradually decomposed, and more silver particles are reduced and gradually bonded together. This leads to the as formed silver particles on the surface of fiber to become more and more compact. When the irradiation time is increased to $5 \mathrm{~h}$, the silver particles are compactly packed together and enabling them to be connected well. This kind of structure effectively improves the conductivity of silver fiber. In addition to the silver particles, the residual carbon-based polymer material was filled in the gap of fibers, thereby further enhancing its flexibility. In fact, the reduction time can be decreased by increasing the power and number of UV lamps. For verifying this hypothesis, we repurchased some UV lamps of different powers. When the power of UV light is up to $250 \mathrm{~W}$, the reduction time decreased to $3 \mathrm{~h}$ resulting in the same sheet resistance as obtained from the previous test (power of UV light: $200 \mathrm{~W}$ ). In addition, the surface temperature of the sample can be controlled to below $100^{\circ} \mathrm{C}$ using the UV light. However, the silver fiber is not electrically conductive based on the sintering treatment which is carried out at the $100^{\circ} \mathrm{C}$ and insulation for 2-10 h. The other important advantages of UV reduction are its convenience, low cost, and the fact that it can be used in large-scale preparation of silver fiber mesh. However, using the sintering treatment, the cost will correspondingly increase. The scale of the prepared silver fiber mesh is limited by the dimension of sintering furnace. Moreover, many flexible substrates are not applicable.

Figure $2 \mathrm{~b}$ depicts the XRD patterns for the fibers using different UV irradiation times and with the augmentation of irradiation time, the characteristic peak of silver nitrate and silver nitrite gradually weakened, while the strength of the Ag peak gradually increased. The XRD pattern revealed the characteristic diffraction peaks of $\mathrm{Ag}$ after the fibers were exposed to UV light for $5 \mathrm{~h}(2 \theta$ values of $38.1^{\circ}, 44.3^{\circ}, 64.5^{\circ}$, and $77.4^{\circ}$, corresponding to the (111),
(200), (220) and (311) crystal faces of the face center cubic crystalline silver). The X-ray photoelectron spectroscopy spectrum of silver submicron fiber is shown in Supplementary Fig. S2, which indicates the two main peaks at 368.3 and $374.3 \mathrm{eV}$, corresponding to $\mathrm{Ag} 3 \mathrm{~d} 5 / 2$ and $3 \mathrm{~d} 3 / 2$ binding energies respectively. Supplementary Figure S3 represents the thermogravimetric analysis/differential thermal analysis (TG/DTA) curves of silver precursor fiber, indicating small amount of mass loss before reaching $150^{\circ} \mathrm{C}$. However, after reaching $150^{\circ} \mathrm{C}$, there is a sharp drop of mass, which indicates the existence of undecomposed polymer after UV treatment, which act as the skeleton of silver fibers to improve their flexibility. Figure $2 c$ presents the Raman spectra of silver precursor fibers and the silver fiber. The Raman spectra of silver precursor fibers indicates that there are Raman active modes at $1040 \mathrm{~cm}^{-1}$, which corresponds to $\mathrm{NO}_{3}{ }^{-}$ion, and for silver fibers two amorphous carbon peaks appear at Raman active modes of 1350 and $1570 \mathrm{~cm}^{-1}$, which manifests that the silver fibers exist on the amorphous carbon. Figure $2 \mathrm{~d}$ gives the elemental scanning images of the silver fiber, indicating that elemental silver is evenly distributed among the fibers, the $C$ and $\mathrm{N}$ elements as the skeleton of the fiber are also distributed evenly in the whole silver fiber. In order to study the structure of the silver fibers further, we test the transmission electron microscopy (TEM) image of single silver fiber (Fig. 2e), The high-resolution TEM images represent the adjacent lattice of silver interconnected into a single structure. Moreover, the silver grains show clear lattice fringes, corresponding to their interplanar spacing of $0.24 \mathrm{~nm}$, thereby proving the existence of a good connection between the adjacent lattice of silver fiber. The fiber surface appears to have grown on an amorphous carbon structure, less than $5 \mathrm{~nm}$ thick, which significantly improves the flexibility of silver fibers. The polycrystalline diffraction rings of silver are shown in the 
a
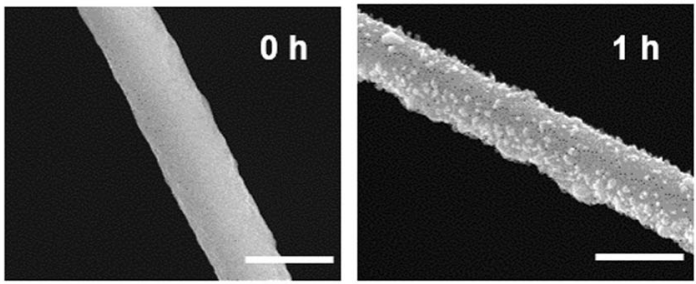

b

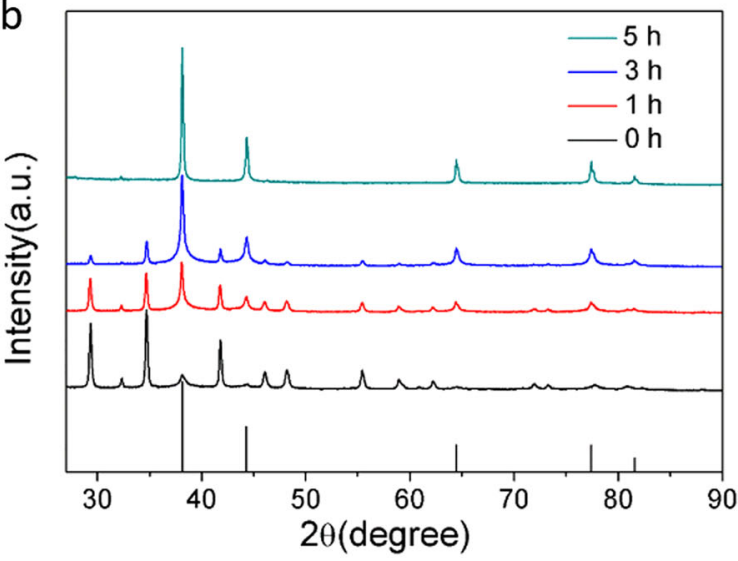

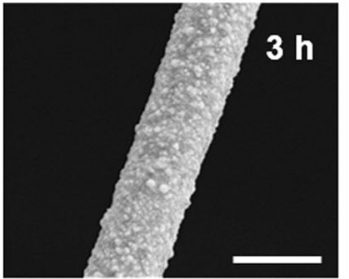
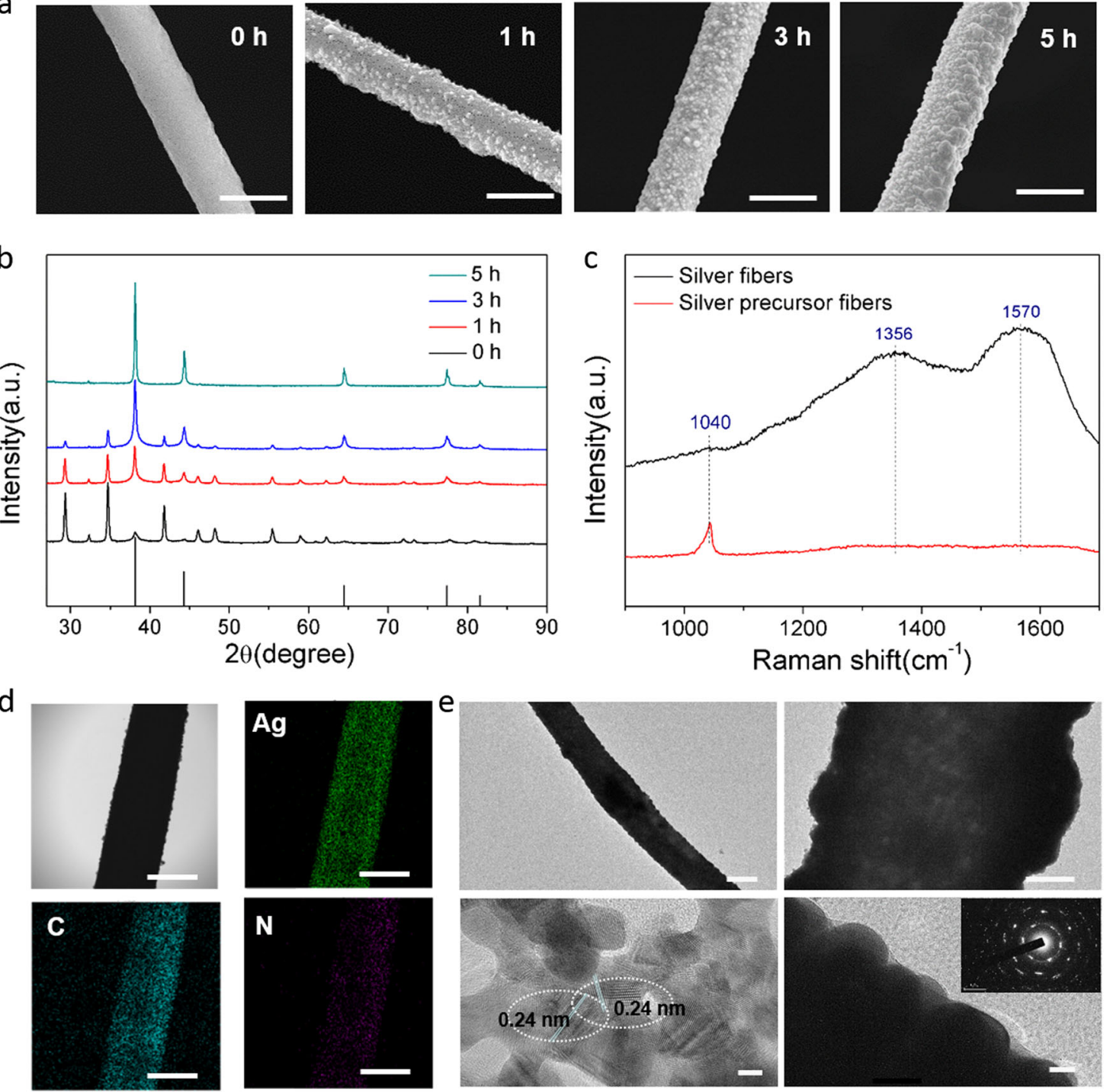

Fig. 2 Reaction process and reaction mechanism of silver precursor fibers turn into the silver fibers. a SEM images of the single fiber after different UV irradiation time (scale bar: $1 \mu \mathrm{m}$ ). b The XRD patterns of silver fibers in different UV irradiation time. c The Raman spectrums of silver precursor fibers and the silver fibers (UV irradiation for $5 \mathrm{~h}$ ). $\mathbf{d}$ Element scanning images of the silver fibers (scale bar: $500 \mathrm{~nm}$ ). e TEM image of single silver fiber (scale bar: $0.5,0.2 \mu \mathrm{m}, 5,20 \mathrm{~nm}$ )

embedded image. Supplementary Figure S4 provides the Auger electron spectroscopy pattern of silver submicron fibers, wherein the different curves represent the composition of the samples corresponding to different depths from the fiber surface (from top: $0,90,180,360 \mathrm{~nm}$ ), which testified the presence of polymer skeleton in silver fiber.

Figure 3a shows the chart presenting the sheet resistance as a function of different transparency of silver fiber mesh measured at a wavelength of $550 \mathrm{~nm}$, indicating that the photoelectric properties of the silver fiber mesh are relatively better compared to the transparent electrodes based solution-processed AgNWs, ${ }^{33}$ graphene-based electrode, ${ }^{13} \mathrm{ITO}^{34} \mathrm{CNT}^{6}$ and Ag nanotrough. ${ }^{28}$ In the case of different sheet resistance, the spacing between the silver fiber mesh is different, specifically, when the average diameter of fibers is 1 micron; the relation between sheet resistances and average spacing of silver fiber mesh as shown in the Supplementary Figure S5. The silver submicron fiber mesh was transferred onto a polyethylene terephthalate (PET) substrate to test its stability in the presence of bending corresponding to different bending radii $(10,9,7,5,2,1 \mathrm{~mm})$ or repeatedly bending the samples up to 1000 times. Once the bending radius of the silver fibers mesh was gradually decreases to $1 \mathrm{~mm}$, the sheet resistance of silver fiber mesh increased by $41 \%$. However, for the copper films subjected to a bending radius of $5 \mathrm{~mm}$, the corresponding sheet resistance increased to $1500 \%$ (Fig. 3b). After the silver fibers mesh was bent for 1000 times (silver fiber mesh is attached to the PET substrate, the bending radius is maintained at $7 \mathrm{~mm}$ ), the sheet resistance of silver fiber mesh increased by $120 \%$, while the sheet resistance of the copper films increased to $1500 \%$ when the number of bending was 500 times (Fig. 3c). Figure $3 \mathrm{~d}$ shows the relationship between the sheet resistance and the stretching quantity of the silver fiber mesh and the copper film. (The silver fiber mesh is attached to a polydimethylsiloxane (PDMS) film of $4 \mathrm{~mm}$ thick). It is clear that when the silver fiber mesh is stretched to $130 \%$ of its original length, the sheet resistance is increased up to $120 \%$, while the sheet resistance of copper films increases to $15,000 \%$ after it is stretched to $115 \%$ of its original length. The free-standing, highly transparent and flexible silver submicron fibers mesh is depicted in Supplementary Video S1. The SEM image of the curved fiber mesh and the TEM image of a folded silver fiber indicates that there is no sign of fracture post bending (Fig. 3e). In order to enhance the toughness 

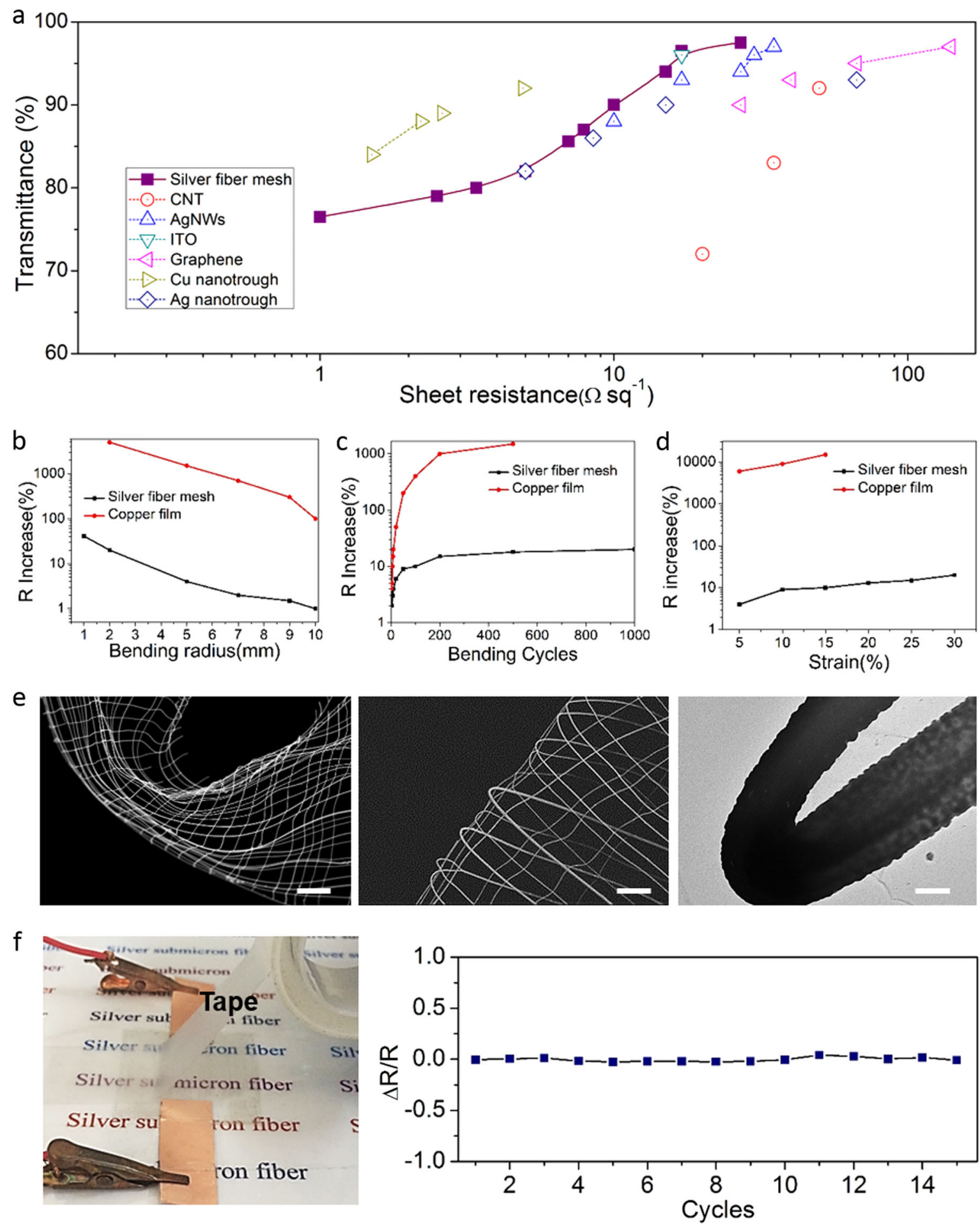

Fig. 3 Photoelectric and toughness studies of silver fiber mesh. a Diagrammatic representation of transparency of different silver fiber mesh as a function of the sheet resistance depicted $550 \mathrm{~nm}$. b Transfer of silver submicron fiber mesh onto a PET substrate to test their stability corresponding different bending radii $(10 \mathrm{~mm}, 9 \mathrm{~mm}, 7 \mathrm{~mm}, 5 \mathrm{~mm}, 2 \mathrm{~mm}, 1 \mathrm{~mm})$. c Plot between times of bending silver fiber mesh versus the corresponding sheet resistance. (The bending radius is at $7 \mathrm{~mm}$ ). d Change in resistance as a function of uniaxial strain for silver fiber mesh and the copper film (the silver mesh is attached to PDMS substrate of $4 \mathrm{~mm}$ thickness). e SEM image of the curved fiber mesh and the TEM image of a folded fiber (scale bar: $100 \mu \mathrm{m}, 20 \mu \mathrm{m}, 500 \mathrm{~nm}$ ). f Stability test of sheet resistance for the silver fiber mesh after being repeatedly fixed with an adhesive tape

and strength of silver fiber mesh, we seal one of the sides of the silver fiber mesh on the PET substrate with a heat-sealing apparatus. The hot melt adhesive: ethylene-vinyl acetate enables the silver fiber mesh to be firmly attached on to the PET substrate.
Figure $3 f$ depicts the results of the stability test of sheet resistance for the silver fiber mesh sealed on the PET substrate after repeatedly attaching with the adhesive tape. The sheet resistance essentially remained stable after repeated tape adhesion for 15 


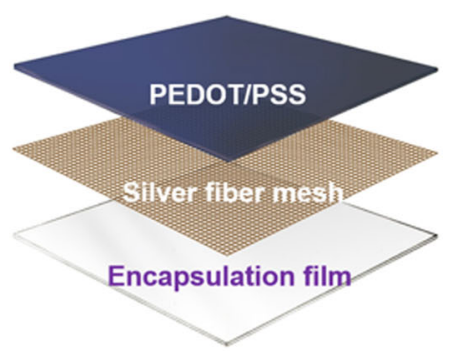

C

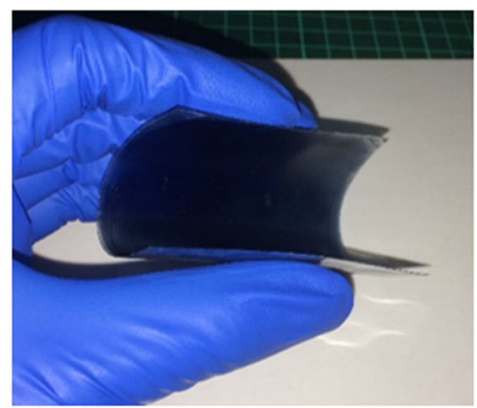

e

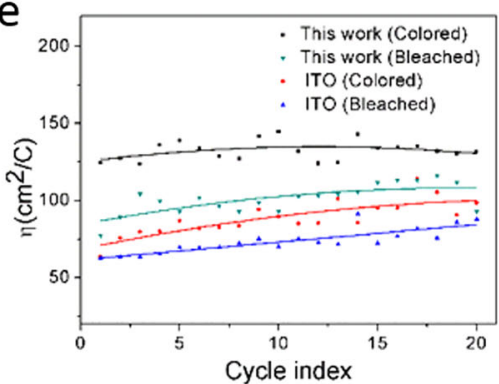

h

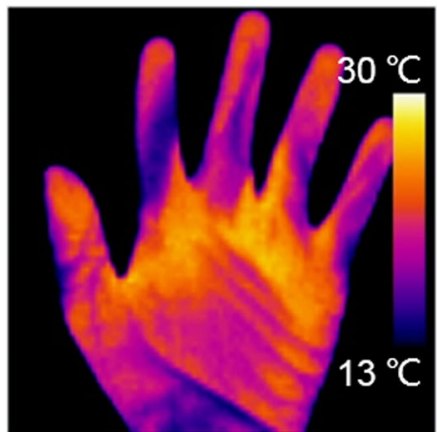

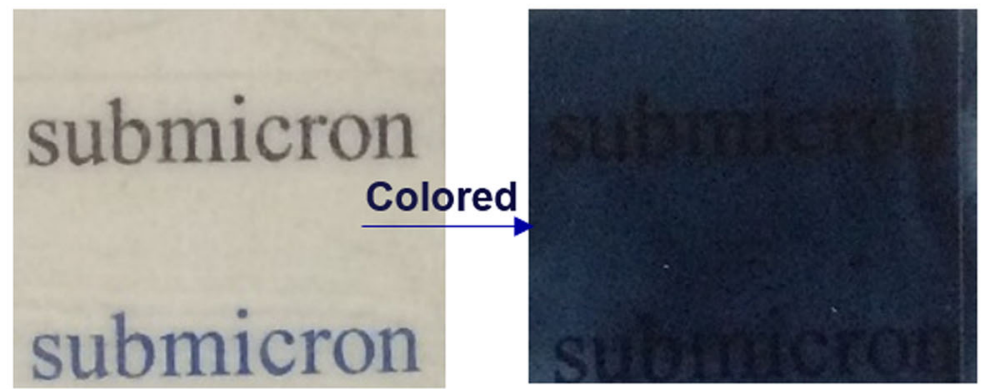
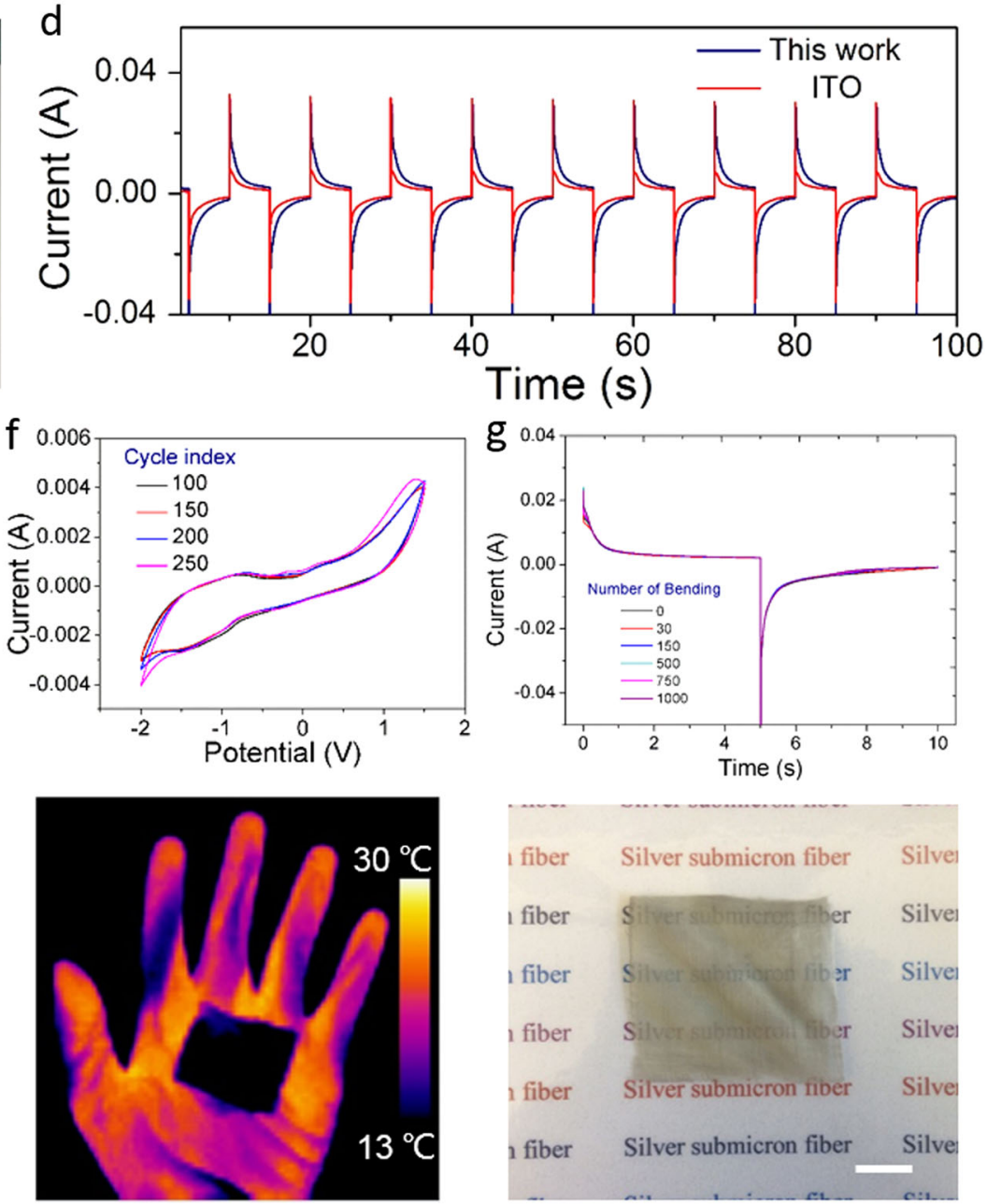

Fig. 4 Pictorial depiction of various applications of silver fiber mesh. a Structural chart depicting the preparation process of electrochromic smart window. $\mathbf{b}$ Images of before and after being colored. c Flexibility for the films after being colored. $\mathbf{d}$ I-t curve corresponding to the sample obtained in this work and ITO. e The electrochromic efficiency of colored and bleached samples obtained from this work shows better than ITO. $\mathbf{f}$ Cyclic voltammetric curve of different cycles $(100,150,200,250)$. $g$ Stability test of the electrochromic smart window after being subjected to bending for up to 1000 times. h Pictorial representation of infrared stealth thin film based on silver fiber mesh (scale bar: $1 \mathrm{~cm}$ )

times. Supplementary Figure S6 represents the stability test of silver submicron fibers/PET substrate in acidic and alkaline conditions, which in turn indicates good resistance stability of silver fiber mesh/PET substrate under acidic or alkaline environment.

At present, ITO as a relatively mature material of transparent electrode, displays relatively excellent photoelectric properties, and is widely used in the industry. However, the large brittleness of the ITO sheets hinders their application in flexible or curved substrates, whereas transparent electrodes based on nanofibers can be conveniently attached with flexible substrates. The change in sheet resistance is insignificant despite repeated bending. In recent years, electrochromic devices have been widely used as a novel energy-saving devices. Their properties of reversible color and transparency can reduce indoor consumption of energy, and effectively solve the problem of light pollution. Increasing number of practical environmental solutions require the flexible, even foldable electrochromic materials. Based on this observation, we use silver fiber mesh as a transparent electrode to prepare flexible electrochromic windows. Figure 4a presents a schematic diagram for the preparation of flexible electrochromic smart window. It is composed of three-layered materials, which consists of an encapsulation film, silver fiber mesh and PEDOT/PSS film. The images of before and after the process of discoloration are shown in Fig. $4 \mathrm{~b}$, which manifest that the film has an obvious change in 
transparency before and after being colored. In addition, the electrochromic smart window still possesses excellent flexibility (Fig. 4c). Figure 4d depicts the I-t curve of the silver fiber mesh and commercial ITO (as a reference), which indicates that our electrochromic smart window has a short response time, suggesting a higher transfer rate of electron as compared to the ITO. Figure 4e shows that the electrochromic efficiency of colored and bleached substrates for our work is better than ITO. The cyclic voltammetry curve of different cycles $(100,150,200,250)$ are shown in Fig. 4f, which indicate that the number of electrons transferred is basically steady after 250 cycles. Figure $4 \mathrm{~g}$ depicts the stability test of the electrochromic smart window after being subjected to bending for 1000 times, and the number of electrons transferred is substantially coincident.

In order to explore more applications of the fiber mesh, infrared stealth studies were performed. Figure $4 \mathrm{~h}$ shows an infrared image of infrared stealth thin film placed on the hand, where the infrared rays are barely radiated on the area of the palm. The corresponding image of the sample is shown in the figure on the right, which indicates that the film still retains certain amount of transparency and breathability. These characteristics enable them to be have great potential for application in practical infrared shielding environment.

\section{DISCUSSION}

In conclusion, the silver fiber mesh with prominent photoelectric properties was obtained by woven mesh of extra-long silver precursor fiber subjected to UV treatment. The as obtained silver fiber possesses micrometer patterning capacity, and can be prepared on a large-scale at room temperature. The silver fiber mesh not only has excellent photoelectric performance, but also displays outstanding flexibility and toughness. In addition, the use of flexible substrates greatly increases the value of practical applications of transparent electrode. The UV irradiation reserves the polymer skeleton of silver fibers, which further enhances their flexibility and toughness. Flexible electrochromic smart window and infrared stealth film are prepared based the silver fiber mesh. The process of continuous draw spinning along with UV treatment opens new avenues for the preparation of ultralong metal submicron fibers at room temperature. We believe that the electronic products based on extra-long silver submicron fiber can be applied to real industrial production in the near future.

\section{METHODS}

\section{Chemicals}

Silver nitrate (99.8\%, Beijing Chemical Works), Polyvinylpyrrolidone (PVP, $M w=1,300,000$, Alfa Aesar), Acetonitrile (99.0\%, Aladdin Industrial Coorporation).

The preparation methods of the extra-long silver fibers and silver fiber mesh

Preparation of silver precursor solution. Usually, the solution of silver precursor fiber needs to be prepared in advance. Firstly, $0.25 \mathrm{~g}$ of PVP and $1.6 \mathrm{~g} \mathrm{AgNO}_{3}$ were added into a glass bottle containing $2 \mathrm{ml}$ of acetonitrile and stirred to form homogeneous solution, continuing to mix the solution evenly, followed by quiescence until there are no bubbles left in the solution.

A demonstration for the preparation of silver precursor fibers. The prepared solution was pumped into a syringe of 1-ml capacity, the syringe was loaded on to the syringe pump, and the discharge rate of the solution was $0.01 \mathrm{ml} / \mathrm{h}$, the substrate rotational speed was $100 \mathrm{r} / \mathrm{min}$, and the substrate forward speed was $3 \mathrm{ml} / \mathrm{h}$, finally forming a uniform array of extra-long silver precursor fiber. The different fiber spacing of the silver fiber mesh can be prepared by reasonably adjusting the rotational speed and forward speed of the substrate.
The preparation process of silver fiber mesh. The prepared silver precursor fiber mesh was placed in a quartz glass box located in the anoxic condition and covered with a lid to prevent oxidation of the silver in air during the UV irradiation. Next the quartz glass box was placed under the UV lamp at a distance of $5 \mathrm{~cm}$, and expose the fibers in UV lamp for $5 \mathrm{~h}$. Finally the silver fiber mesh was taken out from the quartz glass box.

The details about the bending test of silver fiber mesh. The silver fiber mesh was attached to a PET substrate, and the thickness of the copper film (as reference) was kept at $300 \mathrm{~nm}$. For values of different bending radii, the corresponding increase of sheet resistance was recorded. In the case where the bending radius remains a constant $(0.7 \mathrm{~mm})$ while the number of bending times is increased, the corresponding increase of sheet resistance is recorded.

The preparation and test details of electrochromic films PEDOT: PSS composite was spun coated on the silver submicron fiber mesh attached on the PET substrate by using a spin coater (KW-4V, Chemat Technology, America). (spin-coating speed of $2000 \mathrm{rpm}$ and spin-coating time of $30 \mathrm{~s}$ ), then dried in an oven at $70^{\circ} \mathrm{C}$ for $3 \mathrm{~h}$. The experiment for studying electrochromic properties was performed using an electrochemical workstation (CHI 760D, CH Instrument, China) in a two-electrode configuration, where carbon paper used as the counter electrode and lithium perchlorate was used as the electrolyte. The stability test of electrochromic performance after bending was tested via the $1-t$ curve. The multi-potential step method was used to collect data on current corresponding to the process of discoloration and bleaching process of two electrodes at a speed of $0.8 \mathrm{~V} / \mathrm{s}$.

\section{Characterizations}

The surface morphology of the silver submicron fibers was observed through field-emission scanning electron microscopy (LEO-1530). The crystal structure and the materials composition were studied by X-ray diffraction spectroscopy (Rigaku), at a scanning speed is $8 \% \mathrm{~min}$, where the source of radiation is Cu Ka. The Raman spectroscopy technique was used to verify the presence of amorphous carbon in silver fibers with Raman spectrometer (HORIBA Jobin YVON), the wavelength of the Raman laser used in our measurement is $532 \mathrm{~nm}$, and the corresponding power of laser is $250 \mathrm{~W}$. The crystallization of silver fibers and the morphology of silver particles along with their microstructure characterization were measured by a transmission electron microscope (JEOL-2010). Element surface scan image was measured using an energy dispersive spectrometer. The ST$2258 \mathrm{C}$ multifunctional digital four-point probe tester was used to study the sheet resistance of silver fiber mesh. The transparency of different samples of silver fiber mesh was measured by UV-vis spectro-pho-tometer (UV2600 ), and the wavelength range of the measurement was from 400 to $800 \mathrm{~nm}$, A blank glass substrate was used as the reference sample. The TG/ DTA performance was tested by synchronous thermal analyzer (STA 449 F3). The qualitative analysis and valence of the elements in the silver fibers were obtained by X-ray photoelectron spectrometer (Escalab 250Xi), The Scanning Auger Nanoprobe (PHI 710) was used to test the composition of the inside elementary of silver fiber. The infrared image was made by Thermal Imager (DT-980 CEM).

\section{Data availability}

The data supporting the findings of this study are available within the paper [and its Supplementary Information files], or it is also available from the corresponding author upon reasonable request.

\section{ACKNOWLEDGEMENTS}

This study was supported by the National Basic Research of China (Grants 2015CB932500), National Natural Science Foundations of China (Grant 51661135025 and 51522207).

\section{AUTHOR CONTRIBUTIONS}

X.B. and S.L. contributed equally to this work. H.W. and X.B designed the study; X.B. and S. L. synthesized the specimens and performed the analysis. All authors analyzed the data and discussed the results. 


\section{ADDITIONAL INFORMATION}

Supplementary information accompanies the paper on the npj Flexible Electronics website (https://doi.org/10.1038/s41528-017-0016-7).

Competing interests: The authors declare that they have no competing financial interests.

Publisher's note: Springer Nature remains neutral with regard to jurisdictional claims in published maps and institutional affiliations.

\section{REFERENCES}

1. Ye, S., Rathmell, A. R., Chen, Z., Stewart, I. E. \& Wiley, B. J. Metal nanowire networks: the next generation of transparent conductors. Adv. Mater. 26, 6670-6687 (2014).

2. Zhu, R. et al. Fused silver nanowires with metal oxide nanoparticles and organic polymers for highly transparent conductors. ACS nano 5, 9877-9882 (2011).

3. Park, M. et al. Highly stretchable electric circuits from a composite material of silver nanoparticles and elastomeric fibres. Nat. Nanotechnol. 7, 803-809 (2012).

4. De, S. et al. Silver nanowire networks as flexible, transparent, conducting films: extremely high DC to optical conductivity ratios. ACS nano 3, 1767-1774 (2009).

5. $\mathrm{Yu}, \mathrm{Z}$. et al. Highly flexible silver nanowire electrodes for shape-memory polymer light-emitting diodes. Adv. Mater. 23, 664-668 (2011).

6. Hecht, D. S., Hu, L. \& Irvin, G. Emerging transparent electrodes based on thin films of carbon nanotubes, graphene, and metallic nanostructures. Adv. Mater. 23, 1482-1513 (2011).

7. Lee, Y. et al. Versatile metal nanowiring platform for large-scale nano- and optoelectronic devices. Adv. Mater. 28, 9109-9116 (2016).

8. Scheibel, T. et al. Conducting nanowires built by controlled self-assembly of amyloid fibers and selective metal deposition. Proc. Natl. Acad. Sci. USA 100, 4527-4532 (2003).

9. Zeng, W. et al. Fiber-based wearable electronics: a review of materials, fabrication devices, and applications. Adv. Mater. 26, 5310-5336 (2014).

10. Lee, J. et al. Highly conductive and flexible fiber for textile electronics obtained by extremely low-temperature atomic layer deposition of Pt. NPG Asia Mater. 8, e331 (2016).

11. $\mathrm{Wu}, \mathrm{H}$. et al. Electrospun metal nanofiber webs as high-performance transparent electrode. Nano Lett. 10, 4242-4248 (2010).

12. Deb, S. K. et al. Stand-alone photovoltaic-powered electrochromic smart window. Electrochim. Acta 46, 2125-2130 (2001).

13. Bae, S. et al. Roll-to-roll production of 30 -inch graphene films for transparent electrodes. Nat. Nanotechnol. 5, 574-578 (2010).

14. Hu, L., Wu, H. \& Cui, Y. Metal nanogrids, nanowires, and nanofibers for transparent electrodes. MRS Bull. 36, 760-765 (2011).

15. Li, J. et al. Healable capacitive touch screen sensors based on transparent composite electrodes comprising silver nanowires and a furan/maleimide diels-alder cycloaddition polymer. ACS Nano 8, 12874-12882 (2014).

16. Yan, $\mathrm{H}$. et al. Programmable nanowire circuits for nanoprocessors. Nature 470 240-244 (2011).

17. $\mathrm{Yu}, \mathrm{S}$. et al. Area-selective lift-off mechanism based on dual-triggered interfacial adhesion switching: highly facile fabrication of flexible nanomesh electrode. ACS Nano 11, 3506-3516 (2017)

18. Dai, X. et al. Ultra-smooth glassy graphene thin films for flexible transparent circuits. Sci. Adv. 2, e1601574 (2016)
19. Takei, K. et al. Nanowire active-matrix circuitry for low-voltage macroscale artificial skin. Nat. Mater. 9, 821-826 (2010).

20. Lai, Y. C. et al. Electric eel-skin-inspired mechanically durable and super-stretchable nanogenerator for deformable power source and fully autonomous conformable electronic-skin applications. Adv. Mater. 28, 10024-10032 (2016).

21. Wu, H., Huang, Y., Xu, F., Duan, Y. \& Yin, Z. Energy harvesters for wearable and stretchable electronics: from flexibility to stretchability. Adv. Mater. 28, 9881-9919 (2016).

22. Zhao, Y. \& Huang, X. Mechanisms and materials of flexible and stretchable skinsensors. Micromachines 8, 69 (2017).

23. Wang, X. et al. Recent progress in electronic skin. Adv. Sci. (Weinh) 2, 1500169 (2015).

24. Ma, M. et al. Self-powered artificial electronic skin for high-resolution pressure sensing. Nano Energy 32, 389-396 (2017).

25. Yu, Z., Li, L., Zhang, Q., Hu, W. \& Pei, Q. Silver nanowire-polymer composite electrodes for efficient polymer solar cells. Adv. Mater. 23, 4453-4457 (2011).

26. Lipomi, D. J. et al. Skin-like pressure and strain sensors based on transparent elastic films of carbon nanotubes. Nat. Nanotechnol. 6, 788-792 (2011).

27. Hu, L., Kim, H. S., Lee, J.-Y., Peumans, P. \& Cui, Y. Scalable coating and properties of transparent, flexible, silver nanowire electrodes. ACS Nano 4, 2955-2963 (2010).

28. $\mathrm{Wu}, \mathrm{H}$. et al. A transparent electrode based on a metal nanotrough network. Nat Nanotechnol. 8, 421-425 (2013).

29. Lee, J. et al. Very long Ag nanowire synthesis and its application in a highly transparent, conductive and flexible metal electrode touch panel. Nanoscale 4 6408-6414 (2012).

30. Lee, J. G. et al. Production of flexible transparent conducting films of self-fused nanowires via one-step supersonic spraying. Adv. Funct. Mater. 27, 1602548 (2017).

31. Tokuno, T. et al. Fabrication of silver nanowire transparent electrodes at room temperature. Nano Res. 4, 1215-1222 (2011).

32. Bai, X. et al. Continuous draw spinning of extra-long silver submicron fibers with micrometer patterning capability. Nano Lett. 17, 1883-1891 (2017).

33. Guo, F. et al. High-performance semitransparent perovskite solar cells with solution-processed silver nanowires as top electrodes. Nanoscale 7, 1642-1649 (2015).

34. Leem, D. S. et al. Efficient organic solar cells with solution-processed silver nanowire electrodes. Adv. Mater. 23, 4371-4375 (2011).

(i) Open Access This article is licensed under a Creative Commons Attribution 4.0 International License, which permits use, sharing, adaptation, distribution and reproduction in any medium or format, as long as you give appropriate credit to the original author(s) and the source, provide a link to the Creative Commons license, and indicate if changes were made. The images or other third party material in this article are included in the article's Creative Commons license, unless indicated otherwise in a credit line to the material. If material is not included in the article's Creative Commons license and your intended use is not permitted by statutory regulation or exceeds the permitted use, you will need to obtain permission directly from the copyright holder. To view a copy of this license, visit http://creativecommons. org/licenses/by/4.0/.

(c) The Author(s) 2018 